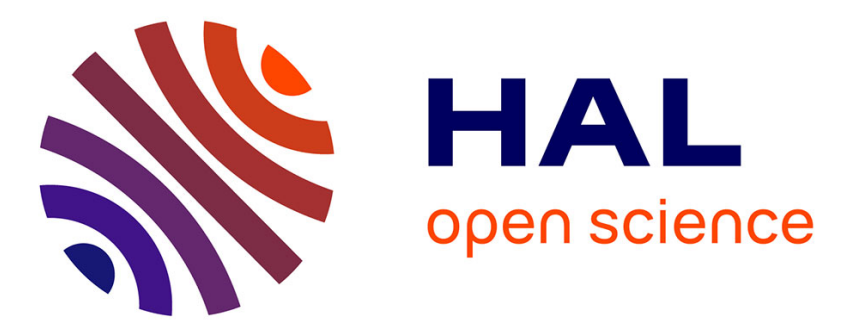

\title{
Pick and release of micro-objects: an actuation-free method to change the conformity of a capillary contact
}

Antonio Iazzolino, Youness Tourtit, Adam Chafai, Tristan Gilet, Pierre Lambert, Loïc Tadrist

\section{- To cite this version:}

Antonio Iazzolino, Youness Tourtit, Adam Chafai, Tristan Gilet, Pierre Lambert, et al.. Pick and release of micro-objects: an actuation-free method to change the conformity of a capillary contact. 2019 International Conference on Manipulation, Automation and Robotics at Small Scales (MARSS), Jul 2019, Helsinki, Finland. pp.1-6, 10.1109/MARSS.2019.8860972 . hal-03222228

\section{HAL Id: hal-03222228 \\ https://hal.science/hal-03222228}

Submitted on 10 May 2021

HAL is a multi-disciplinary open access archive for the deposit and dissemination of scientific research documents, whether they are published or not. The documents may come from teaching and research institutions in France or abroad, or from public or private research centers.
L'archive ouverte pluridisciplinaire HAL, est destinée au dépôt et à la diffusion de documents scientifiques de niveau recherche, publiés ou non, émanant des établissements d'enseignement et de recherche français ou étrangers, des laboratoires publics ou privés. 


\title{
Pick and release of micro-objects: an actuation-free method to change the conformity of a capillary contact.
}

\author{
Antonio Iazzolino ${ }^{1}$, Youness Tourtit ${ }^{1,2}$, Adam Chafaï ${ }^{2}$, Tristan Gilet $^{1}$, Pierre Lambert $^{2}$ and Loïc Tadrist ${ }^{1}$
}

\begin{abstract}
We propose a new 3D printed capillary gripper equipped with a textured surface for actuation-free release. The gripper classically picks up micro-objects exploiting the capillary forces induced by a liquid bridge. Micro-objects are released by decreasing the volume of this bridge through evaporation. This latter can be either natural or speeded up by a heating source (IR laser or Joule effect). The volume reduction changes the topography of the contact (called here conformity) between the gripper and the object. We analyze the gripper performance and rationalize the release mechanism by defining the concept of contact conformity in a capillary context.
\end{abstract}

\section{INTRODUCTION}

Most of the common strategies to handle objects are not suitable at sub-millimeter scale. Vacuum grippers, friction grippers [1] are still predominant for pick-and-place and watch craft industries. The constant miniaturization of objects makes the handling process more and more difficult, because of the associated miniaturization of grippers and the difficulty to manage the positioning and handling of micro-objects without damaging them. These industries also have to comply with increasingly stringent standards on the reliability of the micro-objects they assemble.

Alternative handling mechanisms have been developed for small, fragile and soft objects. There are almost as many handling mechanisms as physical principles developing attractive or restoring forces. Some of them allow to avoid impacts and lacks of conformity, such as smart actuated microgel grippers [2], optical tweezers [3], grippers based on liquid-solid transition (catch-and-block strategy), Bernoulli airflow principle or acoustic levitation [1].

Capillary gripping is another promising handling mechanism that consists in taking benefits of capillary forces, dominant over gravity at the sub-millimeter scale [4]. These forces would have been interfering with other micromanipulation strategies. In the case of grippers, capillary forces are generated by a liquid meniscus binding the gripper and the object to handle. Their application to micro-assembly has been studied in [4] and [5]. Capillary forces result from a tension applied on the gas-liquid-solid triple line interface and the Laplace pressure jump across the interface between gas and liquid. This differential pressure is proportional to the

*Authors thank FNRS financial support through research project T.0050.16 (Bioinspired passive liquid dispensing), FRIA grant, CHAR. RECH.-1.B423.18 (Tadrist L.) and FNRS GEQ - 3D microstructuration and microengineering of surfaces with 3 photons lithography (2014-2016) (Nanoscribe GT Photonics, co-funding ULB/FNRS Grant UG01415F)

${ }^{1}$ Microfluidics Lab, University of Liege, Liège, Belgium, loic.tadrist@uliege. be

${ }^{2}$ TIPs (Tranfers, Interfaces \& Processes), Université Libre de Bruxelles, Bruxelles, Belgium, pierre.lambertaulb.ac.be curvature of the liquid meniscus. The primary idea of using capillary forces as a gripping principle is dated back to 1998 [6] and 2002 [7]. Lambert et al. [8] have demonstrated the feasibility of handling small watch components, namely the sub-millimeter balls of bearings. Since then, many solutions for capillary force control for gripping applications have been developed. Force control represents a major challenge for capillary handling since it establishes the ability to pick, to maintain and especially to release the handled objects.

Different release mechanisms have been considered in previous studies, see Table I. Release mechanisms may be sorted out in two main categories. The first involves applying an additional force to the handled part in order to break the liquid bridge. For example, meniscus shearing is studied in [8], while [9] explores a release by means of the rotation of the handled micro-part induced by the compression under uneven micrometer-sized pillars. The additional force can also be developed by inertia, e.g. in [10] where a large vertical deceleration is applied to the gripper, or in [11] and [12] where vibrations are used. The rupture of the meniscus has also been achieved by generating a higher attraction force on the substrate where the release is expected, e.g. through the addition of glue [10].

The second category of solutions consists in releasing brittle micro-objects without applying additional efforts on them. These solutions are based on the decrease of capillary forces. Bark [6] suggests the injection of gas in order to change the internal pressure in the meniscus. Decreasing capillary forces can also be achieved by monitoring the wettability: capillary forces are decreased thank to electrowetting in [13], [14] and [15]. An elastic gripper is stretched in order to modify its wettability in [16]. Based on Israelachvili's model [17], [18] and [19] have investigated the change of curvature of their grippers, also known as conformity (see Section IV), in order to decrease capillary forces and therefore to release objects. Thermal stimulation can also lead to the decrease of capillary forces by evaporation of the meniscus. This solution is studied in [20] where this evaporation is coupled to the generation of capillary forces on the substrate.

A reliable, accurate, harmless and fast release mechanism is indeed necessary for industrial applications. An Ipulse M20 pick-and-place machine from Yamaha would for instance place one chip every $0.15 \mathrm{~s}$ with 4 picking heads. But contrary to inexpensive micro-objects which are massproduced and which can be processed in fast assembly, the micro-manipulation of brittle objects must give priority to the integrity of the components.

In line with the second category of release mechanisms, 
TABLE I

CENSUS OF RELEASE MECHANISMS FOR CAPILLARY GRIPPERS

\begin{tabular}{|c|c|c|c|c|c|}
\hline Ref. & Release mechanism & $\begin{array}{l}\text { Release duration } \\
\text { depending on }\end{array}$ & $\begin{array}{c}\text { Minimal released } \\
\text { weight }\end{array}$ & $\begin{array}{l}\text { Size and weight of tested } \\
\text { objects }\end{array}$ & $\begin{array}{l}\text { Component possibly } \\
\text { damaged by }\end{array}$ \\
\hline$[8]$ & Meniscus shearing & $\begin{array}{l}\text { Mechanical limits } \\
\text { of actuators * }\end{array}$ & - & $\begin{array}{c}\mathrm{S}: \mathrm{D}=0.5 \mathrm{~mm} \\
\mathrm{~W}=3 \cdot 8 \cdot 10^{-3} \mathrm{mN}\end{array}$ & Mechanical efforts * \\
\hline [9] & Rotation of handled object & $\begin{array}{l}\text { Mechanical limits } \\
\text { of actuators * }\end{array}$ & - & C: $1 \times 0.5 \times 0.5 \mathrm{~mm}^{3}$ & Mechanical efforts * \\
\hline [10] & Glue on substrate & Fluid motion * & - & - & $\begin{array}{l}\text { Chemical contamina- } \\
\text { tion * }\end{array}$ \\
\hline [10] & $\begin{array}{l}\text { Inertia - Vertical } \\
\text { deceleration }\end{array}$ & $\begin{array}{l}\text { Mechanical limits } \\
\text { of actuators * }\end{array}$ & $\begin{array}{l}\text { Depending on applied } \\
\text { accelerations * }\end{array}$ & - & Mechanical efforts * \\
\hline $\begin{array}{l}{[11]} \\
{[12]}\end{array}$ & Inertia - Vibrations & $\begin{array}{l}\text { Mechanical limits } \\
\text { of actuators * }\end{array}$ & $\begin{array}{l}\text { Depending on } \\
\text { amplitude and } \\
\text { frequency } *\end{array}$ & $\begin{array}{c}\text { S: } \mathrm{D}=0.04-0.2 \mathrm{~mm} \\
\mathrm{~W}=0.35 .10^{-6}-43.4 .10^{-6} \mathrm{mN} \\
\mathrm{C}: 0.5 \times 0.5 \times 0.28 \mathrm{~mm}^{3} \\
\mathrm{~W}=1.63 .10^{-3} \mathrm{mN}^{-3} \\
\mathrm{C}: 1 \times 1 \times 0.52 \mathrm{~mm}^{3} \\
\mathrm{~W}=12.1 .10^{-3} \mathrm{mN}\end{array}$ & Mechanical efforts * \\
\hline$[6]$ & Injection of gas & Fluid motion * & - & $\begin{array}{c}\mathrm{C}: 4.2 \times 4.2 \times 0.5 \mathrm{~mm} 3 \\
\mathrm{~W}=0.2 \mathrm{mN}\end{array}$ & None $*$ \\
\hline $\begin{array}{l}{[13]} \\
{[14]} \\
{[15]}\end{array}$ & Electrowetting & Fluid motion * & $77 \mathrm{mN}$ & $\mathrm{W}=77.10^{-3}-136.10^{-3} \mathrm{mN}$ & Electric current $*$ \\
\hline [16] & Stretching of the gripper & Fluid motion * & - & $\begin{array}{c}\mathrm{S}: \mathrm{D}=0.5-2.85 \mathrm{~mm} \\
\mathrm{~W}=3-373 \mathrm{mN} \\
\text { Microscrews: } 0.5-2.5 \mathrm{~mm} \\
\mathrm{~W}=28-310 \mathrm{mN}\end{array}$ & None $*$ \\
\hline $\begin{array}{l}{[18]} \\
{[19]}\end{array}$ & Change in conformity & Fluid motion * & $0.47 \mathrm{mN}$ & $\mathrm{W}=0.295-2.18 \mathrm{mN} *$ & None $*$ \\
\hline [20] & $\begin{array}{l}\text { Evaporation and capillary } \\
\text { forces on substrate }\end{array}$ & Fluid motion * & No limit * & $S: D=0.005-0.06 \mathrm{~mm}$ & None * \\
\hline
\end{tabular}

S: Sphere (diameter is given), C: Cuboid (lengths are given), W: Weight of object(s), *: Estimations made by us

this paper explores an actuation-free release mechanism for capillary grippers based on a change of the conformity in the contact area. This change is allowed by surface texturing and bridge volume reduction through evaporation. This article first presents the setup and methods to study this new release strategy. The experimental results are then compared to models. In particular, the concept of contact conformity is defined in the context of capillary gripping. Finally, conclusions and perspectives are discussed in section V.

\section{MATERIAL AND METHODS}

\section{A. Description of the gripper}

The gripper used for all the experiments is a cone covered with spine-like microstructures called barbules (Fig. $1 b)$. The gripper is $3 \mathrm{D}$-printed with the two-photon-initiated polymerization-based stereolithography system Nanoscribe, with a $200 \mathrm{~nm}$ resolution. The gripper material is IP-L 780 UV-curable photoresin. The main cone has a height of $700 \mu \mathrm{m}$ and an aperture angle of $140^{\circ}$. The barbules are also cones, of half aperture $12.5^{\circ}$, with a tilt angle respective to the cone surface of $50^{\circ}$. The perpendicular distance from the surface of the cone to the head of the tip is $30 \mu \mathrm{m}$ and the distance between two barbules is $50 \mu \mathrm{m}$. The barbule density is 50 barbules $/ \mathrm{mm}^{2}$.

\section{B. Description of the experimental setup}

The experimental setup is described in Fig. 1. The gripper is mounted on a 3 degrees-of-freedom translation stage.
High-precision mechanical stages are used for an accurate positioning of the gripper in the $(x, y)$ plane. The vertical motion of the gripper is controlled with a linear actuator (MTS25) with a resolution of $0.5 \mu \mathrm{m}$.

A droplet of approximately $10 \mu \mathrm{L}$ of purified water is deposited on a metallic substrate. To ensure reproducibility, the substrate is cleaned with ethanol and a new droplet is deposited before each experiment. The gripper is first vertically moved (z-axis) downwards the droplet to form a liquid bridge. It is then moved upwards, which stretches and breaks the liquid bridge. A liquid volume of $1.9 \pm 0.4 \mu \mathrm{L}$ is left on the gripper. Reduced volumes of liquid, ([0.7, 0.4, $0.1] \mu \mathrm{L}$ ), were obtained by drying the initial liquid bridge with an IR laser $(1455 \mathrm{~nm})$ at $491 \mathrm{~mW}$ (for [15, 20, 30] s respectively).

Idealized millimeter-sized objects, each consisting of a spherical cap on top of a vertical cylinder of radius $1 \mathrm{~mm}$, have been 3D-printed with a resin of density $\rho=$ $1.17 \mathrm{~kg} . \mathrm{m}^{-3}$ (VeroWhitePlusRGD 835). Three cap radii were tested $\left(R_{1}=625 \mu \mathrm{m}, R_{2}=950 \mu \mathrm{m}\right.$, and $\left.R_{3}=1250 \mu \mathrm{m}\right)$ and the mass of the objects was varied from $1 \mathrm{mg}$ to $30 \mathrm{mg}$ by changing the length of the cylinder. The objects are initially placed on a micro-structured planar surface. The microstructures are again conical tips, which purpose is to minimize solid-solid contact forces.

The gripper is moved downward until a capillary bridge is formed with the object. The object then follows the gripper: it can be lifted and moved horizontally at the same time as the gripper (see video in supplementary material), 
(a)
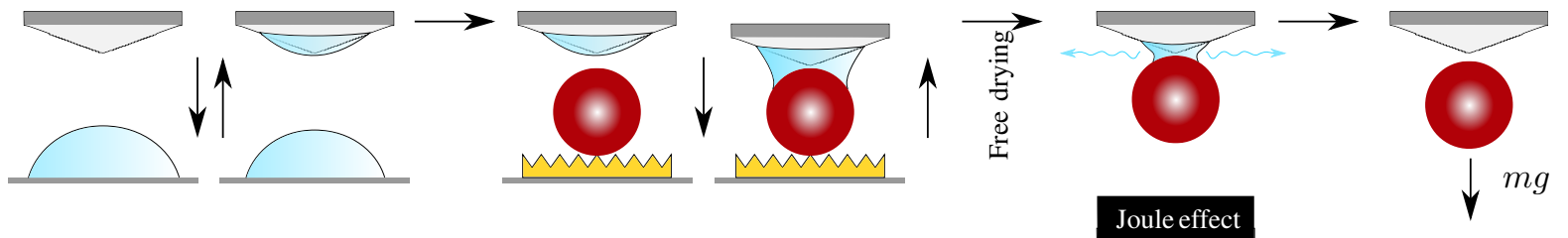

(b)
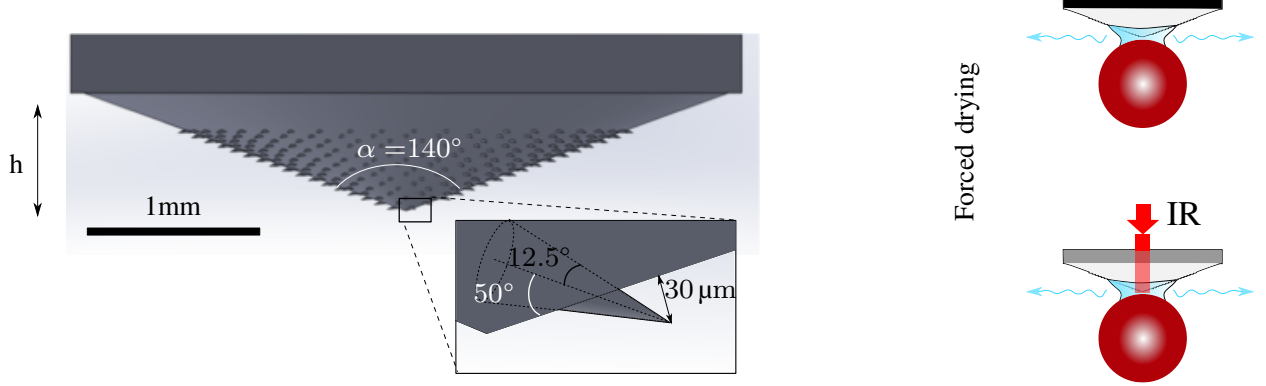

Fig. 1. (a) Schematics of the experimental procedure. (b) CAD geometry of the conical gripper. Close-up on a barbule.

but the strength of this binding has not extensively been characterized experimentally yet.

The water bridge volume is decreased, through either natural or forced evaporation. Two techniques were considered to force evaporation. The first is based on the Joule effect: an electrical current flows in a ceramic heater plate and produces heat. The heat is transferred by conduction from the plate to the gripper then to water. The second technique involves an IR laser with a wavelength of $1455 \mathrm{~nm}$. The gripper made of IP-L $780 \mathrm{UV}$-curable photoresin is indeed transparent to this wavelength. The IR light is efficiently absorbed by water with an absorption coefficient of $2.6 \times 10^{3} \mathrm{~m}^{-1}$. The absorbed energy is turned into heat, which induces water evaporation.

The experiment has been imaged from the side with a camera (Canon EOS 5D Mark III) and a macro lens (Canon-MP-E 65mm f/2.8 1-5x Macro). The resolution is $3.8 \mu \mathrm{m} / \mathrm{pixel}$.

\section{GRIPPING PERFORMANCE}

The ability of this gripper to pick and release millimetersized objects is first characterized. Relevant dimensional parameters are (i) the volume of water $V$ used to pick up the object, (ii) the surface tension of water $\gamma$, (iii) the mass $m$ of the object, (iv) the radius of curvature $R$ of the spherical cap on top of the object, and (v) gravity $g$. An educated guess [21] would reveal that the density $\rho$ of the liquid is not relevant, since the Laplace pressure induced by surface tension is much higher than the hydrostatic pressure inside this sub-millimeter liquid bridge. From these relevant parameters, we can build two dimensionless numbers, namely the Bond number and the length ratio:

$$
B_{o}=\frac{m g}{\gamma V^{1 / 3}} \quad \text { and } \quad r=\frac{V^{1 / 3}}{R} .
$$

The capability of the gripper is summarized in the diagram $\left(B_{o}, r\right)$ of Fig. 2. Three zones are identified: (i) the gripper can pick and release the object, (ii) the gripper can pick the

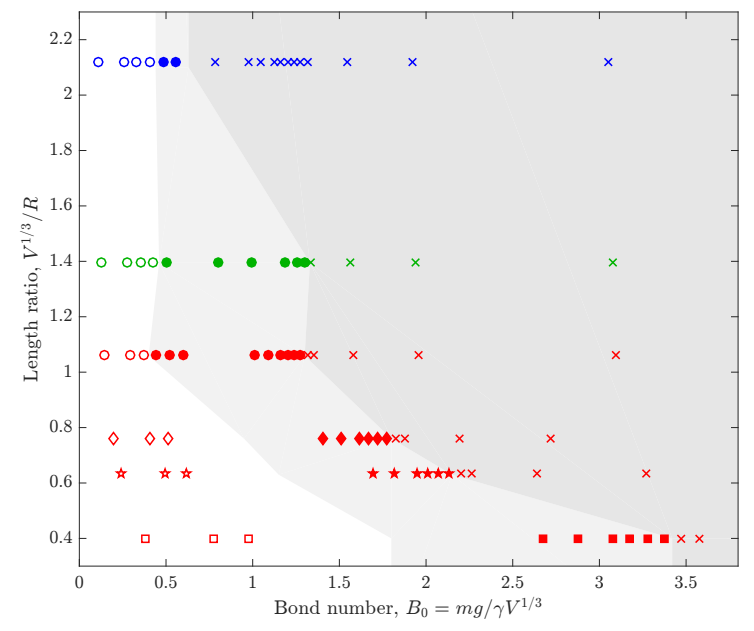

Fig. 2. Diagram of the gripper capability in terms of Bond number $B_{o}$ and length ratio $r$. Three radii of curvature of the object were considered, $R=625 \mu \mathrm{m}$ (blue), $R=950 \mu \mathrm{m}$ (green), $R=1250 \mu \mathrm{m}$ (red). The symbols correspond to the liquid volume, $1.9 \mu \mathrm{L}(\circ), 0.7 \mu \mathrm{L}(\diamond), 0.4 \mu \mathrm{L}(\star)$ and $0.1 \mu \mathrm{L}(\square)$. Empty symbols and white background indicate the ability of the gripper to pick up the object but the impossibility to release it. Full symbols and light gray background indicate that the gripper can both pick up and release the object. Symbols $\times$ and dark gray background indicate that the gripper could not pick up the object.

object but not release it, and (iii) the gripper cannot pick the object.

The release performance of the evaporative gripper has also been quantified. The relation between the release time and the power used to warm up the liquid is represented in Fig. 3. For both the Joule effect and the laser heating, the release time $\tau$ is observed to be inversely proportional to the applied power $P$.

\section{MOdEL OF THE RELEASE TIME}

The experimental results of Fig. 3 on the evaporation time of the bridge can be rationalized with theoretical 


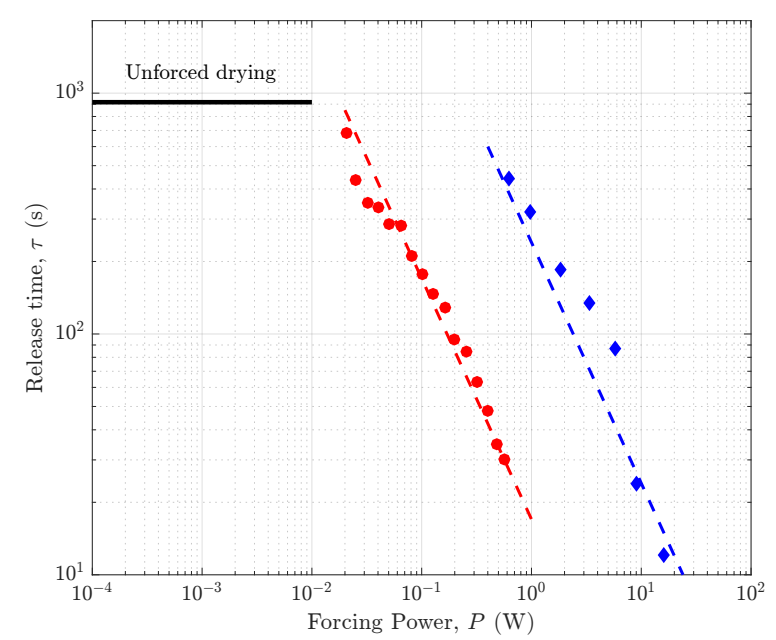

Fig. 3. Release time $\tau$ as a function of the input power $P$ of the heating system (initial bridge volume $1.9 \mu \mathrm{L}$ ). (red) IR laser. (blue) Joule effect. Dotted lines correspond to equations 4 and 7, which confirm the scaling law $\tau \propto 1 / P$. Unforced break-up time is reported as an asymptote (black line) in the log-log representation.

arguments. In the absence of convection, natural evaporation is only driven by the diffusion of water vapour, as initially modelled by Langmuir [24]. As a crude approximation, the liquid bridge is here replaced by a hemispherical droplet of the same volume. If the fine geometry of the bridge is taken into account it will only change the prefactor but not the scalings. The diameter $\delta$ of this hemisphere is then $\delta(t)=(12 V(t) / \pi)^{1 / 3}$. The relative humidity $\varphi$ in the air surrounding the bridge is by definition the ratio of the partial vapor pressure $P_{v}$ and the vapor pressure at saturation $P_{v}^{0}$. It satisfies the Laplace equation for pseudo-steady-state molecular diffusion in spherical coordinates, namely $\nabla^{2} \varphi=$ 0 . If we assume that gradients of $\varphi$ are only radial, the vapor concentration profile is $\varphi(r)=\varphi_{\infty}+\left(1-\varphi_{\infty}\right) \delta(t) / 2 r$, where $r$ is the radial coordinate and $\varphi_{\infty}$ the relative humidity of the room during the experiment. The diffusive mass flux is then calculated as $J=D M_{w} P_{v}^{0}\left(1-\varphi_{\infty}\right) \delta /\left(2 r^{2} R T\right)$ where $D$ is the diffusion coefficient of water vapor in air, $M_{w}$ the molar mass of water, $R$ the ideal gas constant and $T$ the room temperature. A mass balance of water between liquid and gas phases then yields:

$$
\rho \frac{d V}{d t}=-2 \pi r^{2} J
$$

The volume of the liquid bridge then evolves from an initial value $V_{0}$ as

$$
V(t)=V_{0}\left(1-\frac{t}{\tau}\right)^{3 / 2} \text { with } \tau=\frac{\left(3 V_{0} / 2 \pi\right)^{2 / 3} \rho R T}{2 D M_{w} P_{v}^{0}\left(1-\varphi_{\infty}\right)}
$$

Complete drying occurs at time $\tau$, which varies as $V_{0}^{2 / 3}$. This time has been measured experimentally $\tau_{\text {exp }}=917 \pm 44 \mathrm{~s}$ and is reported in Fig. 3. The model evaluated with parameter values from Table II predicts $\tau=2150 \mathrm{~s}$, which is larger than in experiments but still of the same order of magnitude.
The discrepancy can be attributed to the replacement of the bridge geometry by a hemisphere, the assumption of a purely diffusive evaporation, and the fact that some liquid may remain at break-up.

When heat is brought to the bridge, the evaporation is not driven by vapor diffusion anymore. For the heating strategy based on the Joule effect, the supplied power $P$ yields an increase of thermal energy $\eta P t$ after time $t$, where the factor $\eta<1$ is the fraction of produced heat that reaches the bridge. This thermal energy is used to first warm up the liquid to $100{ }^{\circ} \mathrm{C}$ (heat capacity) and then to evaporate it (latent heat). As the former requires about 7 times less energy than the latter, it can be neglected in first approximation. The energy balance then yields:

$$
\eta P t=h \rho\left[V_{0}-V(t)\right]
$$

where $h=2.26 \times 10^{6} \mathrm{~J} /(\mathrm{kg} \mathrm{K})$ is the specific latent heat of water. Therefore, the volume decreases linearly with time:

$$
V(t)=V_{0}\left(1-\frac{t}{\tau}\right) \text { where } \tau=\frac{\rho h V_{0}}{\eta P}
$$

is the time of complete evaporation of the bridge. The yield $\eta$ in equation 5 is estimated to $1.8 \%$ by fitting on experimental data (Fig. 3).

When the bridge is evaporated by laser-induced heating, the amount of absorbed energy depends on the liquid thickness through the Beer-Lambert law. Therefore, the yield now depends on the thickness of the bridge, which scales as $\beta V^{1 / 3}$ where $\beta$ is an unknown geometrical coefficient. For thin films, the absorbance is $\alpha \beta V^{1 / 3}$ where $\alpha$ is the attenuation coefficient of the electromagnetic wave sent by the laser (here $1455 \mathrm{~nm}$ ) in water. The energy balance then yields

$$
\left(\eta \alpha \beta V^{1 / 3}\right) P=-\rho h \frac{\mathrm{d} V}{\mathrm{~d} t} .
$$

The temporal evolution of liquid volume is then,

$$
V(t)=V_{0}\left(1-\frac{t}{\tau}\right)^{3 / 2} \quad \text { where now } \quad \tau=\frac{3 \rho h V_{0}^{2 / 3}}{2 \eta \alpha \beta P}
$$

Again, a comparison of this theoretical prediction to experimental data in Fig. 3 indicates that the factor $\eta \beta$ is about $11.8 \%$.

Both models of forced evaporation suggest that the release time is inversely proportional to the input power $P$. However, its dependence to the initial liquid volume $V_{0}$ differs: it is proportional in the case of the Joule-induced evaporation, and less than proportional in the case of laser-induced evaporation. We expect that the use of a larger forcing power might keep reducing the releasing time, especially for the laser method that is not limited by thermal conduction in the gripper.

\section{Discussion}

The contact conformity [22] refers to the shape of the contact, as defined in solid mechanics. There is conformal contact when two solids fit one another similarly to a ball in spherical cup of identical diameter. The meniscus mediated 
TABLE II

PARAMETERS AND CONSTANTS USED IN THE MODELS,

\begin{tabular}{|c|c|c|}
\hline \multicolumn{3}{|c|}{ VARIABLES \& COORDINATES } \\
\hline$P_{v}$ & Water vapour pressure & \\
\hline$\varphi$ & Relative humidity & \\
\hline$V$ & Bridge volume & \\
\hline$\delta$ & Equivalent diameter & \\
\hline$r$ & Radial coordinate & \\
\hline$t$ & time & \\
\hline & EXPERIMENTAL PARAMETERS & VALUE \\
\hline$\varphi_{\infty}$ & Ambient relative humidity & 0.55 \\
\hline$V_{0}$ & Initial bridge volume & 0.5 to $210^{-9} \mathrm{~m}^{3}(\mu \mathrm{L})$ \\
\hline$T$ & Room temperature & $293.15 \mathrm{~K}$ \\
\hline$P$ & Forcing power & 0.1 to $10 \mathrm{~W}$ \\
\hline$m$ & Initial mass of liquid & 0.5 to $210^{-6} \mathrm{~kg}$ \\
\hline$\eta$ & Yield of evaporation process & - \\
\hline & CONSTANTS & VALUE \\
\hline$P_{v}^{0}$ & Saturated water vapor pressure & $2.3410^{3} \mathrm{~Pa}$ \\
\hline$D$ & Diffusion coefficient of water vapor & $2.8210^{-5} \mathrm{~m}^{2} \cdot \mathrm{s}^{-1}$ \\
\hline$M_{w}$ & Water molar mass & $1810^{-3} \mathrm{~kg} \cdot \mathrm{mol}^{-1}$ \\
\hline$R$ & Ideal gas constant & $8.314 \mathrm{~J} . \mathrm{mol}^{-1} \cdot \mathrm{K}^{-1}$ \\
\hline$\rho$ & Water density & $1.010^{3} \mathrm{~kg} \cdot \mathrm{m}^{-3}$ \\
\hline$c_{p}$ & Water specific heat capacity & $4.210^{3} \mathrm{~J} . \mathrm{kg}^{-1} \cdot \mathrm{K}^{-1}$ \\
\hline$h$ & Water specific latent heat & $2.2610^{6} \mathrm{~J}^{\mathrm{kg}} \mathrm{kg}^{-1}$ \\
\hline$\alpha$ & Attenuation coefficient at $1455 \mathrm{~nm}$ & $2.610^{3} \mathrm{~m}^{-1}$ \\
\hline
\end{tabular}

contact between the gripper and the object evolves during the drying process. At the beginning, the barbules are submerged by the liquid and do not play any other role than possibly pinning the triple line during liquid uptake. As the liquid volume is reduced, the conformity shifts from a cone-sphere contact to a barbule-sphere contact. By reducing the volume of liquid, we simply change the scale at which the surface of the gripper is seen.

For a capillary gripper, a contact is said conformal when it maximizes the capillary force, which is somehow related to the geometrical conformity borrowed from contact mechanics but not only [5]. The capillary force is maximized when two solids have a large contact area and a large curvature where the Laplace pressure jump occurs.

To simplify the discussion, we describe the contact geometry only with the radii of curvature $R_{1}$ and $R_{2}$ of the two objects. We also further assume that the contact angle $\theta$ is the same on both solids. The capillary force $F$ reads [23],

$$
F=4 \pi \gamma \lambda \cos \theta
$$

where the conformity $\lambda$ is defined as

$$
\lambda=\frac{R_{1} R_{2}}{R_{1}+R_{2}} .
$$

One note that when the contact conformity is perfect, i.e. when two objects fit perfectly, $R_{1}=-R_{2}$ and the conformity is infinite. Such configuration would maximize the attractive capillary forces. Conversely, if the first object has a much smaller radius of curvature than the second, the conformity $\lambda \sim R_{1}$ is consequently small.

This concept may help to understand how our gripper works. The object has a radius of curvature $R_{1}$. To grab it, we use a wet cone for which the typical radius of curvature $R_{2}$ depends on the liquid volume: the more liquid is attached to the cone, the larger is $R_{2}$. During picking up, conformity should be maximized, which suggests to use a large liquid volume (i.e. $R_{2} \gg R 1$ ). The picking conformity is driven by the component radius $\lambda=R_{1}$.

As the bridge dries, $R_{2}$ is reduced and so is $\lambda$. In rare cases this might be sufficient to detach the object, however we noticed during preliminary tests that the object usually sticks on the side of a smooth cone where curvature is finite. On the textured gripper, barbules finally come into contact with the object as the liquid dries up. If the radius of barbule is smaller than the one of the object $R_{b} \ll R_{1}$, the conformity is strongly reduced, which would now be of the order of the barbule radius $\lambda \sim R_{b}$. With the textured gripper, the conformity at picking $\left(\lambda \sim R_{1}\right)$ is larger than conformity at releasing $\left(\lambda \sim R_{b}\right)$, making detachment possible. Consequently, the object may detach from the gripper as soon as the conformity leads to a capillary force smaller that the object weight.

This capillary gripper releases objects without applying additional efforts. We can therefore imagine using this technique on brittle objects in a pick-and-place context. In the case of the Joule effect stimulation, the thermal diffusion that occurs through the gripper would limit the effect of power increase. The release time would be rather long compared to those used in industry. However, we expect the release time to be strongly reduced, down to a fraction of second, if the IR-laser power is increased. Here, the release time would potentially compare with those of industrial grippers. Even if millimeter sized objects were considered as a first step, we expect the sizes and weights of handled objects to decrease along with the size of the gripper. The precision of the stereolithography printing technique, i.e. $200 \mu \mathrm{m}$, may indeed allow us to consider smaller objects. We also considered barbules for the griper surface texture as a first step. Any other shape giving a small conformity to the contact (i.e. having a large radius of curvature, such as spines or hairs) may be efficient. No optimization of the gripper design has been done. In our mind, the combination of the conformity at the gripper scale and at the texture scale can be strongly improved. For instance, a gripper with a radius curvature matching the opposite object radius of curvature and the textures having a very large radius of curvature compared to the object size would make a very efficient gripper. The wetability of the gripper has not been varied in this study but is known to play an important role in the gripping of microobjects. We expect that hydrophobic surface treatments may improve the release capability of the gripper. One of the most important next steps of this work will be to complement the phase-diagram of Fig. 2, by measuring the force developed by the gripper as a function of the liquid volume and the temperature.

In summary, we designed a 3D-printed capillary gripper based on a change of conformity of the contact for pick-up and release. The contact conformity is changed by evaporation of the liquid bridge. It switches scale, from the large cone to the small barbules. The evaporation may be forced by Joule effect or laser absorption to reduce the release time. 
Finally we use the capillary conformity as an efficient proxy to explain the pick and release mechanism.

\section{ACKNOWLEDGMENT}

Authors warmly thank F.N. Piñan Basualdo for letting them use his laser set-up.

\section{REFERENCES}

[1] G. Fantoni et al., "Grasping devices and methods in automated production processes," CIRP Annals, vol. 63, pp. 679-701, 2014.

[2] H. Jia et al., "Universal Soft Robotic Microgripper," Small, in press.

[3] D. G. Grier, "A revolution in optical manipulation," Nature, in press.

[4] P. Lambert, Surface tension effects in microsystems, Springer, 2013, p. 4.

[5] P. Lambert, Capillary forces in microassembly, Springer, 2007.

[6] C. Bark, T. Binnenbose, G . Vogele, T. Weisener, M. Widmann, "Gripping with low viscosity fluids," Proceedings of the IEEE Micro Electro Mechanical Systems, pp. 301-305, January 1998.

[7] H. Grutzeck, L. Kiesewetter, "Downscaling of grippers for micro assembly," Microsystem Technologies, vol. 8, pp. 27-31, 2002.

[8] P. Lambert, Frank Seigneur, Sandra Koelemeijer, Jacques Jacot "A case study of surface tension gripping: the watch bearing," J. Micromech. Microeng., in press.

[9] M. Cavaiani, Sam Dehaeck, Youen Vitry, Pierre Lambert, "Multi-scale 3D printed capillary gripper," International Conf. on Manipulation, Automation and Robotics at Small Scales, October 2018.

[10] M. Dafflon, "Préhenseur, conditions et stratégies pour une micromanipulation de précision", Lausanne, $\mathrm{PhD}$ Thesis, $\mathrm{CH}$ : École Polytechnique Fédérale de Lausanne, 2008.

[11] Z. Fan, W. Rong, L. Wang and L. Sun - "A single-probe capillary microgripper induced by dropwise condensation and inertial release," J. Micromech. Microeng., in press.
[12] Z. Fan, L. Wang, W. Rong, L. Sun, "Dropwise condensation on a hydrophobic probe-tip for manipulating micro-objects," Applied physics letters, in press.

[13] A. Vasudev, A. Jagtiani, L. Du, J. Zhe, "A low-voltage droplet microgripper for micro-object manipulation," J. Micromech. Microeng., in press.

[14] M. Decre, R. Wolf, Manipulation of objects with fluid droplets, US patent WO 2006/0226013 A1.

[15] F. Apoorva, R. Maccurdy, H. Lipson, System and methods for electrowetting based pick and place, WO patent 2014/014892 A2

[16] G. Fantoni, H. Nørgaard Hansen, M. Santochi, ”A new capillary gripper for mini and macro parts," CIRP Annals, vol. 62, pp. 17-20, 2013.

[17] J. N. Israelchvili, Intermolecular and surface forces , 3rd ed., Academic Press, 2011, p. 458.

[18] Biganzoli, I. Fassi, and C. Pagano, "Development of a gripping system based on capillary force,'IEEE International Symposium on Assembly and Task Planning, vol. 2005, pp. 36-40, July 2005.

[19] C. Pagano, L Zanoni, I. Fassi, F. Jovane, "Micro-assembly: Design and analysis of a gripper based on capillary force," CIRP International Seminar on Assembly Systems, pp. 165-170, November 2006.

[20] S. Uran, R. Šafarič and B. Bratina, "Reliable and accurate release of micro-sized objects with a gripper that uses the capillary-force method," Micromachines, in press.

[21] C.J. Gommes and T. Gilet, "Combine Dimensional Analysis with Educated Guessing." Chemical Engineering Progress 114.2: 49-54, 2018.

[22] J. M. Skotheim and L. Mahadevan. "Soft lubrication: the elastohydrodynamics of nonconforming and conforming contacts." Physics of Fluids 17.9 : 092101, 2005.

[23] P. Lambert, " A contribution to microassembly: a study of capillary forces as a gripping principle", PhD Thesis, Université Libre de Bruxelles, 2005.

[24] I. Langmuir, "The evaporation of small spheres." Physical review 12.5 : $368,1918$. 